\title{
On total mean curvatures of foliated half-lightlike submanifolds in semi-Riemannian manifolds
}

\author{
Fortuné Massamba*iD, Samuel Ssekajja \\ School of Mathematics, Statistics and Computer Science, University of KwaZulu-Natal, Private Bag X01, \\ Scottsville 3209 South Africa
}

\begin{abstract}
We derive total mean curvature integration formulas of a three co-dimensional foliation $\mathcal{F}^{n}$ on a screen integrable half-lightlike submanifold, $M^{n+1}$ in a semi-Riemannian manifold $\bar{M}^{n+3}$. We give generalized differential equations relating to mean curvatures of a totally umbilical half-lightlike submanifold admitting a totally umbilical screen distribution, and show that they are generalizations of those given by [K. L. Duggal and B. Sahin, Differential geometry of lightlike submanifolds, Frontiers in Mathematics, Birkhäuser Verlag, Basel, 2010].
\end{abstract}

Mathematics Subject Classification (2010). 53C25, 53C40, 53C50

Keywords. half-lightlike submanifold, Newton transformation, foliation and mean curvature

\section{Introduction}

The rapidly growing importance of lightlike submanifolds in semi-Riemannian geometry, particularly Lorentzian geometry, and their applications to mathematical physics-like in general relativity and electromagnetism motivated the study of lightlike geometry in semi-Riemannian manifolds. More precisely, lightlike submanifolds have been shown to represent different black hole horizons (see [4] and [6] for details). Among other motivations for investing in lightlike geometry by many physicists is the idea that the universe we are living in can be viewed as a 4-dimensional hypersurface embedded in $(4+m)$ dimensional spacetime manifold, where $m$ is any arbitrary integer. There are significant differences between lightlike geometry and Riemannian geometry as shown in [4] and [6], and many more references therein. Some of the pioneering work on this topic is due to Duggal-Bejancu [4], Duggal-Sahin [6] and Kupeli [7]. It is upon those books that many other researchers, including but not limited to [3,5,8-11], have extended their theories.

Lightlike geometry rests on a number of operators, like shape and algebraic invariants derived from them, such as trace, determinants, and in general the $r$-th mean curvature $S_{r}$. There is a great deal of work so far on the case $r=1$ (see some in $[4,6]$ and many more) and as far as we know, very little has been done for the case $r>1$. This is partly due to the non-linearity of $S_{r}$ for $r>1$, and hence very complicated to study. A great

\footnotetext{
*Corresponding Author.

Email addresses: massfort@yahoo.fr and Massamba@ukzn.ac.za (F. Massamba), ssekajja.samuel.buwaga@aims-senegal.org (S. Ssekajja)

Received: 07.03.2017; Accepted: 18.03.2019
} 
deal of research on higher order mean curvatures $S_{r}$ in Riemannian geometry has been done with numerous applications, for instance see [2] and [1]. This gap has motivated our introduction of lightlike geometry of $S_{r}$ for $r>1$. In this paper we have considered a halflightlike submanifold admitting an integrable screen distribution, of a semi-Riemannian manifold. On it we have focused on a codimension 3 foliation of its screen distribution and thus derived integral formulas of its total mean curvatures (see Theorems 4.9 and 4.10). Furthermore, we have considered totally umbilical half-lightlike submanifolds, with a totally umbilical screen distribution and generalized Theorem 4.3.7 of [6] (see Theorem 5.2 and its Corollaries). The paper is organized as follows; In Section 2 we summarize the basic notions on lightlike geometry necessary for other sections. In Section 3 we give some basic information on Newton transformations of a foliation $\mathcal{F}$ of the screen distribution. Section 4 focuses on integration formulae of $\mathcal{F}$ and their consequences. In Section 5 we discus screen umbilical half-lightlike submanifolds and generalizations of some well-known results of $[6]$.

\section{Preliminaries}

Let $\left(M^{n+1}, g\right)$ be a two-co-dimensional submanifold of a semi-Riemannian manifold $\left(\bar{M}^{n+3}, \bar{g}\right)$, where $g=\left.\bar{g}\right|_{T M}$. The submanifold $\left(M^{n+1}, g\right)$ is called a half-lightlike if the radical distribution $\operatorname{Rad} T M=T M \cap T M^{\perp}$ is a vector subbundle of the tangent bundle $T M$ and the normal bundle $T M^{\perp}$ of $M$, with rank one. Let $S(T M)$ be a screen distribution which is a semi-Riemannian complementary distribution of $\operatorname{Rad} T M$ in $T M$, and also choose a screen transversal bundle $S\left(T M^{\perp}\right)$, which is semi-Riemannian and complementary to $\operatorname{Rad} T M$ in $T M^{\perp}$. Then,

$$
T M=\operatorname{Rad} T M \perp S(T M), T M^{\perp}=\operatorname{Rad} T M \perp S\left(T M^{\perp}\right) .
$$

We will denote by $\Gamma(\Xi)$ the set of smooth sections of the vector bundle $\Xi$. It is well-known from [4] and [6] that for any null section $E$ of $\operatorname{Rad} T M$, there exists a unique null section $N$ of the orthogonal complement of $S\left(T M^{\perp}\right)$ in $S(T M)^{\perp}$ such that $g(E, N)=1$, it follows that there exists a lightlike transversal vector bundle $\operatorname{lt}(T M)$ locally spanned by $N$. Let $W \in \Gamma\left(S\left(T M^{\perp}\right)\right)$ be a unit vector field, then $\bar{g}(N, N)=\bar{g}(N, Z)=\bar{g}(N, W)=0$, for any $Z \in \Gamma(S(T M))$.

Let $\operatorname{tr}(T M)$ be complementary (but not orthogonal) vector bundle to $T M$ in $T \bar{M}$. Then we have the following decompositions of $\operatorname{tr}(T M)$ and $T \bar{M}$

$$
\begin{aligned}
\operatorname{tr}(T M) & =l \operatorname{tr}(T M) \perp S\left(T M^{\perp}\right), \\
T \bar{M} & =S(T M) \perp S\left(T M^{\perp}\right) \perp\{\operatorname{Rad} T M \oplus l \operatorname{tr}(T M)\} .
\end{aligned}
$$

It is important to note that the distribution $S(T M)$ is not unique, and is canonically isomorphic to the factor vector bundle $T M / \operatorname{Rad} T M$ [4]. Let $P$ be the projection of $T M$ on to $S(T M)$. Then the local Gauss-Weingarten equations of $M$ are the following;

$$
\begin{aligned}
& \bar{\nabla}_{X} Y=\nabla_{X} Y+B(X, Y) N+D(X, Y) W, \\
& \bar{\nabla}_{X} N=-A_{N} X+\tau(X) N+\rho(X) W, \\
& \bar{\nabla}_{X} W=-A_{W} X+\phi(X) N, \\
& \nabla_{X} P Y=\nabla_{X}^{*} P Y+C(X, P Y) E, \\
& \nabla_{X} E=-A_{E}^{*} X-\tau(X) E,
\end{aligned}
$$

for all $E \in \Gamma(\operatorname{Rad} T M), N \in \Gamma(l \operatorname{tr}(T M))$ and $W \in \Gamma\left(S\left(T M^{\perp}\right)\right)$, where $\nabla$ and $\nabla^{*}$ are induced linear connections on $T M$ and $S(T M)$, respectively, $B$ and $D$ are called the local second fundamental forms of $M, C$ is the local second fundamental form on $S(T M)$. Furthermore, $\left\{A_{N}, A_{W}\right\}$ and $A_{E}^{*}$ are the shape operators on $T M$ and $S(T M)$ respectively, and $\tau, \rho, \phi$ and $\delta$ are differential 1 -forms on $T M$. Notice that $\nabla^{*}$ is a metric connection 
on $S(T M)$ while $\nabla$ is generally not a metric connection. In fact, $\nabla$ satisfies the following relation

$$
\left(\nabla_{X} g\right)(Y, Z)=B(X, Y) \lambda(Z)+B(X, Z) \lambda(Y),
$$

for all $X, Y, Z \in \Gamma(T M)$, where $\lambda$ is a 1 -form on $T M$ given $\lambda(\cdot)=\bar{g}(\cdot, N)$. It is well-known from [4] and [6] that $B$ and $D$ are independent of the choice of $S(T M)$ and they satisfy

$$
B(X, E)=0, \quad D(X, E)=-\phi(X), \forall X \in \Gamma(T M) .
$$

The local second fundamental forms $B, D$ and $C$ are related to their shape operators by the following equations

$$
\begin{aligned}
& g\left(A_{E}^{*} X, Y\right)=B(X, Y), \quad \bar{g}\left(A_{E}^{*} X, N\right)=0, \\
& g\left(A_{W} X, Y\right)=\varepsilon D(X, Y)+\phi(X) \lambda(Y), \\
& g\left(A_{N} X, P Y\right)=C(X, P Y), \quad \bar{g}\left(A_{N} X, N\right)=0, \\
& \bar{g}\left(A_{W} X, N\right)=\varepsilon \rho(X), \quad \text { where } \varepsilon=\bar{g}(W, W),
\end{aligned}
$$

for all $X, Y \in \Gamma(T M)$. From equations (2.11) we deduce that $A_{E}^{*}$ is $S(T M)$-valued, self-adjoint and satisfies $A_{E}^{*} E=0$. Let $\bar{R}$ denote the curvature tensor of $\bar{M}$, then

$$
\begin{aligned}
\bar{g}(\bar{R}(X, Y) P Z, N) & =g\left(\left(\nabla_{X} A_{N}\right) Y, P Z\right)-g\left(\left(\nabla_{Y} A_{N}\right) X, P Z\right) \\
& +\tau(Y) C(X, P Z)-\varepsilon \tau(X) C(Y, P Z)\{\rho(Y) D(X, P Z) \\
& -\rho(X) D(Y, P Z)\}, \quad \forall X, Y, Z \in \Gamma(T M) .
\end{aligned}
$$

A half-lightlike submanifold $(M, g)$ of a semi-Riemannian manifold $\bar{M}$ is said to be totally umbilical [6] if on each coordinate neighborhood $\mathcal{U}$ there exist smooth functions $\mathcal{H}_{1}$ and $\mathcal{H}_{2}$ on $l \operatorname{tr}(T M)$ and $S\left(T M^{\perp}\right)$ respect such that

$$
B(X, Y)=\mathcal{H}_{1} g(X, Y), \quad D(X, Y)=\mathcal{H}_{2} g(X, Y), \quad \forall X, Y \in \Gamma(T M) .
$$

Furthermore, when $M$ is totally umbilical then the following relations follows by straightforward calculations

$$
A_{E}^{*} X=\mathcal{H}_{1} P X, \quad P\left(A_{W} X\right)=\varepsilon \mathcal{H}_{2} P X, \quad D(X, E)=0, \quad \rho(E)=0,
$$

for all $X, Y \in \Gamma(T M)$.

Next, we suppose that $M$ is a half-lightlike submanifold of $\bar{M}$, with an integrable screen distribution $S(T M)$. Let $M^{\prime}$ be a leaf of $S(T M)$. Notice that for any screen integrable half-lightlike $M$, the leaf $M^{\prime}$ of $S(T M)$ is a co-dimension 3 submanifold of $\bar{M}$ whose normal bundle is $\{\operatorname{Rad} T M \oplus l \operatorname{tr}(T M)\} \perp S\left(T M^{\perp}\right)$. Now, using (2.4) and (2.7) we have

$$
\bar{\nabla}_{X} Y=\nabla_{X}^{*} Y+C(X, P Y) E+B(X, Y) N+D(X, Y) W
$$

for all $X, Y \in \Gamma\left(T M^{\prime}\right)$. Since $S(T M)$ is integrable, then its leave is semi-Riemannian and hence we have

$$
\bar{\nabla}_{X} Y=\nabla_{X}^{*^{\prime}} Y+h^{\prime}(X, Y), \quad \forall X, Y \in \Gamma\left(T M^{\prime}\right),
$$

where $h^{\prime}$ and $\nabla^{*^{\prime}}$ are second fundamental form and the Levi-Civita connection of $M^{\prime}$ in $\bar{M}$. From (2.18) and (2.19) we can see that

$$
h^{\prime}(X, Y)=C(X, P Y) E+B(X, Y) N+D(X, Y) W
$$

for all $X, Y \in \Gamma\left(T M^{\prime}\right)$. Since $S(T M)$ is integrable, then it is well-known from [6] that $C$ is symmetric on $S(T M)$ and also $A_{N}$ is self-adjoint on $S(T M)$ (see Theorem 4.1.2 for details). Thus, $h^{\prime}$ given by (2.20) is symmetric on $T M^{\prime}$.

Let $L \in \Gamma\left(\{\operatorname{Rad} T M \oplus l \operatorname{tr}(T M)\} \perp S\left(T M^{\perp}\right)\right)$, then we can decompose $L$ as

$$
L=a E+b N+c W
$$


for non-vanishing smooth functions on $\bar{M}$ given by $a=\bar{g}(L, N), b=\bar{g}(L, E)$ and $c=$ $\varepsilon \bar{g}(L, W)$. Suppose that $\bar{g}(L, L)>0$, then using $(2.21)$ we obtain a unit normal vector $\widehat{W}$ to $M^{\prime}$ given by

$$
\widehat{W}=\frac{1}{\bar{g}(L, L)}(a E+b N+c W)=\frac{1}{\bar{g}(L, L)} L .
$$

Next we define a $(1,1)$ tensor $\mathcal{A}_{\widehat{W}}$ in terms of the operators $A_{E}^{*}, A_{N}$ and $A_{W}$ by

$$
\mathcal{A}_{\widehat{W}} X=\frac{1}{\bar{g}(L, L)}\left(a A_{E}^{*} X+b A_{N} X+c A_{W} X\right),
$$

for all $X \in \Gamma(T M)$. Notice that $\mathcal{A}_{\widehat{W}}$ is self-adjoint on $S(T M)$. Applying $\bar{\nabla}_{X}$ to $\widehat{W}$ and using equations (2.23) (2.4) and (2.11)-(2.13), we have

$$
g\left(\mathcal{A}_{\widehat{W}} X, P Y\right)=-\bar{g}\left(\bar{\nabla}_{X} \widehat{W}, P Y\right), \quad \forall X, Y \in \Gamma(T M) .
$$

Let $\nabla^{* \perp}$ be the connection on the normal bundle $\{\operatorname{Rad} T M \oplus l \operatorname{tr}(T M)\} \perp S\left(T M^{\perp}\right)$. Then from (2.24) we have

$$
\bar{\nabla}_{X} \widehat{W}=-\mathcal{A}_{\widehat{W}} X+\nabla_{X}^{* \perp} \widehat{W}, \quad \forall X \in \Gamma(T M)
$$

where

$$
\begin{aligned}
\nabla_{X}^{* \perp} \widehat{W} & =-\frac{1}{\bar{g}(L, L)} X(\bar{g}(L, L)) \widehat{W}+\frac{1}{\bar{g}(L, L)}[\{X(a)-a \tau(X)\} E \\
& +\{X(b)+b \tau(X)+c \phi(X)\} N+\{X(c)+a D(X, E)+b \rho(X)\} W] .
\end{aligned}
$$

Example 2.1. Let $\bar{M}=\left(\mathbb{R}_{1}^{5}, \bar{g}\right)$ be a semi-Riemannian manifold, where $\bar{g}$ is of signature $(-,+,+,+,+)$ with respect to canonical basis $\left(\partial x_{1}, \partial x_{2}, \partial x_{3}, \partial x_{4}, \partial x_{5}\right)$, where $\left(x_{1}, \cdots, x_{5}\right)$ are the usual coordinates on $\bar{M}$. Let $M$ be a submanifold of $\bar{M}$ and given parametrically by the following equations

$$
\begin{array}{r}
x_{1}=\varphi_{1}, \quad x_{2}=\sin \varphi_{2} \sin \varphi_{3}, \quad x_{3}=\varphi_{1}, \quad x_{4}=\cos \varphi_{2} \sin \varphi_{3}, \\
x_{5}=\cos \varphi_{3}, \text { where } \varphi_{2} \in[0,2 \pi] \text { and } \varphi_{3} \in(0, \pi / 2) .
\end{array}
$$

Then we have $T M=\operatorname{span}\left\{E, Z_{1}, Z_{2}\right\}$ and $l \operatorname{tr}(T M)=\operatorname{span}\{N\}$, where

$$
\begin{aligned}
& E=\partial x_{1}+\partial x_{3}, \quad Z_{1}=\cos \varphi_{3} \partial x_{2}-\sin \varphi_{2} \sin \varphi_{3} \partial x_{5} \\
& Z_{2}=\cos \varphi_{3} \partial x_{4}-\cos \varphi_{2} \sin \varphi_{3} \partial x_{5} \text { and } N=\frac{1}{2}\left(-\partial x_{1}+\partial x_{3}\right) .
\end{aligned}
$$

Also, by straightforward calculations, we have

$$
W=\sin \varphi_{2} \sin \varphi_{3} \partial x_{2}+\cos \varphi_{2} \sin \varphi_{3} \partial x_{4}+\cos \varphi_{3} \partial x_{5} .
$$

Thus, $S\left(T M^{\perp}\right)=\operatorname{span}\{W\}$ and hence $M$ is a half-lightlike submanifold of $\bar{M}$. Furthermore we have $\left[Z_{1}, Z_{2}\right]=\cos \varphi_{2} \sin \varphi_{3} \partial x_{2}-\sin \varphi_{2} \sin \varphi_{3} \partial x_{4}$, which leads to $\left[Z_{1}, Z_{2}\right]=$ $\cos \varphi_{2} \tan \varphi_{3} Z_{1}-\sin \varphi_{2} \tan \varphi_{3} Z_{2} \in \Gamma(S(T M))$. Thus, $M$ is a screen integrable half-lightlike submanifold of $\bar{M}$. Finally, it is easy to see that $A_{N}$ is self-adjoint operator on $S(T M)$.

In the next sections we shall consider screen integrable half-lightlike submanifolds of semi-Riemannian manifold $\bar{M}$ and derive special integral formulas for a foliation of $S(T M)$, whose normal vector is $\widehat{W}$ and with shape operator $\mathcal{A}_{\widehat{W}}$. 


\section{Newton transformations of $\mathcal{A}_{\widehat{W}}$}

Let $\left(\bar{M}^{m+3}, \bar{g}\right)$ be a semi-Riemannian manifold and let $\left(M^{n+1}, g\right)$ be a screen integrable half-lightlike submanifold of $\bar{M}$. Then $S(T M)$ admits a foliation and let $\mathcal{F}$ be a such foliation. Then, the leaves of $\mathcal{F}$ are co-dimension three submanifolds of $\bar{M}$, whose normal bundle is $S(T M)^{\perp}$. Let $\widehat{W}$ be unit normal vector to $\mathcal{F}$ such that the orientation of $\bar{M}$ coincides with that given by $\mathcal{F}$ and $\widehat{W}$. The Levi-Civita connection $\bar{\nabla}$ on the tangent bundle of $\bar{M}$ induces a metric connection $\nabla^{\prime}$ on $\mathcal{F}$. Furthermore, $h^{\prime}$ and $\mathcal{A}_{\widehat{W}}$ are the second fundamental form and shape operator of $\mathcal{F}$. Notice that $\mathcal{A}_{\widehat{W}}$ is self-adjoint on $T \mathcal{F}$ and at each point $p \in \mathcal{F}$ has $n$ real eigenvalues (or principal curvatures) $\kappa_{1}(p), \cdots, \kappa_{n}(p)$. Attached to the shape operator $\mathcal{A}_{\widehat{W}}$ are $n$ algebraic invariants

$$
S_{r}=\sigma_{r}\left(\kappa_{1}, \cdots, \kappa_{n}\right), \quad 1 \leq r \leq n,
$$

where $\sigma_{r}: M^{\prime n} \rightarrow \mathbb{R}$ are symmetric functions given by

$$
\sigma_{r}\left(\kappa_{1}, \cdots, \kappa_{n}\right)=\sum_{1 \leq i_{1}<\cdots<i_{r} \leq n} \kappa_{i_{1}} \cdots \kappa_{i_{r}} .
$$

Then, the characteristic polynomial of $\mathcal{A}_{\widehat{W}}$ is given by

$$
\operatorname{det}\left(\mathcal{A}_{\widehat{W}}-t \mathbb{I}\right)=\sum_{\alpha=0}^{n}(-1)^{\alpha} S_{r} t^{n-\alpha},
$$

where $\mathbb{I}$ is the identity in $\Gamma(T \mathcal{F})$. The normalized $r$-th mean curvature $H_{r}$ of $M^{\prime}$ is defined by

$$
\left.H_{r}=\left(\begin{array}{l}
n \\
r
\end{array}\right)^{-1} S_{r} \text { and } H_{0}=1 \text {. (a constant function } 1\right) .
$$

In particular, when $r=1$ then $H_{1}=\frac{1}{n} \operatorname{tr}\left(\mathcal{A}_{\widehat{W}}\right)$ which is the mean curvature of a $\mathcal{F}$. On the other hand, $H_{2}$ relates directly with the (intrinsic) scalar curvature of $\mathcal{F}$. Moreover, the functions $S_{r}\left(H_{r}\right.$ respectively) are smooth on the whole $M$ and, for any point $p \in \mathcal{F}$, $S_{r}$ coincides with the $r$-th mean curvature at $p$. In this paper, we shall use $S_{r}$ instead of $H_{r}$.

Next, we introduce the Newton transformations with respect to the operator $\mathcal{A}_{\widehat{W}}$. The Newton transformations $T_{r}: \Gamma(T \mathcal{F}) \rightarrow \Gamma(T \mathcal{F})$ of a foliation $\mathcal{F}$ of a screen integrable halflightlike submanifold $M$ of an $(n+3)$-dimensional semi-Riemannian manifold $\bar{M}$ with respect to $A_{\widehat{W}}$ are given by by the inductive formula

$$
T_{0}=\mathbb{I}, \quad T_{r}=(-1)^{r} S_{r} \mathbb{I}+\mathcal{A}_{\widehat{W}} \circ T_{r-1}, \quad 1 \leq r \leq n .
$$

By Cayley-Hamiliton theorem, we have $T_{n}=0$. Moreover, $T_{r}$ are also self-adjoint and commutes with $\mathcal{A}_{\widehat{W}}$. Furthermore, the following algebraic properties of $T_{r}$ are well-known (see [2], [1] and references therein for details).

$$
\begin{aligned}
\operatorname{tr}\left(T_{r}\right) & =(-1)^{r}(n-r) S_{r}, \\
\operatorname{tr}\left(\mathcal{A}_{\widehat{W}} \circ T_{r}\right) & =(-1)^{r}(r+1) S_{r+1}, \\
\operatorname{tr}\left(\mathcal{A}_{\widehat{W}}^{2} \circ T_{r}\right) & =(-1)^{r+1}\left(-S_{1} S_{r+1}+(r+2) S_{r+2}\right), \\
\operatorname{tr}\left(T_{r} \circ \nabla_{X}^{\prime} \mathcal{A}_{\widehat{W}}\right) & =(-1)^{r} X\left(S_{r+1}\right)=(-1)^{r} \bar{g}\left(\nabla^{\prime} S_{r+1}, X\right),
\end{aligned}
$$

for all $X \in \Gamma(T \bar{M})$. We will also need the following divergence formula for the operators $T_{r}$

$$
\operatorname{div}^{\nabla^{\prime}}\left(T_{r}\right)=\operatorname{tr}\left(\nabla^{\prime} T_{r}\right)=\sum_{\beta=1}^{n}\left(\nabla_{Z_{\beta}}^{\prime} T_{r}\right) Z_{\beta},
$$

where $\left\{Z_{1}, \cdots, Z_{n}\right\}$ is a local orthonormal frame field of $T \mathcal{F}$. 


\section{Integration formulas for $\mathcal{F}$}

This section is devoted to derivation of integral formulas of foliation $\mathcal{F}$ of $S(T M)$ with a unit normal vector $\widehat{W}$ given by (2.22). By the fact that $\bar{\nabla}$ is a metric connection then $\bar{g}\left(\overline{\nabla_{\widehat{W}}} \widehat{W}, \widehat{W}\right)=0$. This implies that the vector field $\bar{\nabla}_{\widehat{W}} \widehat{W}$ is always tangent to $\mathcal{F}$. Our main goal will be to compute the divergence of the vectors $T_{r} \bar{\nabla}_{\widehat{W}} \widehat{W}$ and $T_{r} \bar{\nabla}_{\widehat{W}} \widehat{W}+$ $(-1)^{r} S_{r+1} \widehat{W}$. The following technical lemmas are fundamentally important to this paper. Let $\left\{E, Z_{i}, N, W\right\}$, for $i=1, \cdots, n$ be a quasi-orthonormal field of frame of $T \bar{M}$, such that $S(T M)=\operatorname{span}\left\{Z_{i}\right\}$ and $\epsilon_{i}=\bar{g}\left(Z_{i}, Z_{i}\right)$.

Lemma 4.1. Let $M$ be a screen integrable half-lightlike submanifold of $\bar{M}^{n+3}$ and let $M^{\prime}$ be a foliation of $S(T M)$. Let $\mathcal{A}_{\widehat{W}}$ be its shape operator, where $\widehat{W}$ is a unit normal vector to $\mathcal{F}$. Then

$$
\begin{aligned}
\bar{g}\left(\left(\nabla_{X}^{\prime} \mathcal{A}_{\widehat{W}}\right) Y, Z\right) & =\bar{g}\left(Y,\left(\nabla_{X}^{\prime} \mathcal{A}_{\widehat{W}}\right) Z\right), \\
\bar{g}\left(\left(\nabla_{X}^{\prime} T_{r}\right) Y, Z\right) & =\bar{g}\left(Y,\left(\nabla_{X}^{\prime} T_{r}\right) Z\right),
\end{aligned}
$$

for all $X, Y, Z \in \Gamma(T \mathcal{F})$.

Proof. By simple calculations we have

$$
\bar{g}\left(\left(\nabla_{X}^{\prime} \mathcal{A}_{\widehat{W}}\right) Y, Z\right)=\bar{g}\left(\nabla_{X}^{\prime}\left(\mathcal{A}_{\widehat{W}} Y\right), Z\right)-\bar{g}\left(\nabla_{X}^{\prime} Y, \mathcal{A}_{\widehat{W}} Z\right) .
$$

Using the fact that $\nabla^{\prime}$ is a metric connection and the symmetry of $\mathcal{A}_{\widehat{W}}$, (4.1) gives

$$
\bar{g}\left(\left(\nabla_{X}^{\prime} \mathcal{A}_{\widehat{W}}\right) Y, Z\right)=\bar{g}\left(Y, \nabla_{X}^{\prime}\left(\mathcal{A}_{\widehat{W}} Z\right)\right)-\bar{g}\left(Y, \mathcal{A}_{\widehat{W}}\left(\nabla_{X}^{\prime} Z\right)\right) .
$$

Then, from (4.2) we deduce the first relation of the lemma. A proof of the second relation follows in the same way, which completes the proof.

Lemma 4.2. Let $M$ be a screen integrable half-lightlike submanifold of $\bar{M}$ and let $\mathcal{F}$ be a co-dimension three foliation of $S(T M)$. Let $\mathcal{A}_{\widehat{W}}$ be its shape operator, where $\widehat{W}$ is a unit normal vector to $\mathcal{F}$. Denote by $\bar{R}$ the curvature tensor of $\bar{M}$. Then

$$
\begin{aligned}
& \operatorname{div}^{\nabla^{\prime}}\left(T_{0}\right)=0, \\
& \operatorname{div}^{\nabla^{\prime}}\left(T_{r}\right)=\mathcal{A}_{\widehat{W}} \operatorname{div}^{\nabla^{\prime}}\left(T_{r-1}\right)+\sum_{i=1}^{n} \epsilon_{i}\left(\bar{R}\left(\widehat{W}, T_{r-1} Z_{i}\right) Z_{i}\right)^{\prime},
\end{aligned}
$$

where $(\bar{R}(\widehat{W}, X) Z)^{\prime}$ denotes the tangential component of $\bar{R}(\widehat{W}, X) Z$ for $X, Z \in \Gamma(T \mathcal{F})$. Equivalently, for any $Y \in \Gamma(T \mathcal{F})$ then

$$
\bar{g}\left(\operatorname{div}^{\nabla^{\prime}}\left(T_{r}\right), Y\right)=\sum_{j=1}^{r} \sum_{i=1}^{n} \epsilon_{i} \bar{g}\left(\bar{R}\left(T_{r-1} Z_{i}, \widehat{W}\right)\left(-\mathcal{A}_{\widehat{W}}\right)^{j-1} Y, Z_{i}\right) .
$$

Proof. The first equation of the lemma is obvious since $T_{0}=\mathbb{I}$. We turn to the second relation. By direct calculations using the recurrence relation (3.2) we derive

$$
\begin{aligned}
\operatorname{div}^{\nabla^{\prime}}\left(T_{r}\right) & =(-1)^{r} \operatorname{div}^{\nabla^{\prime}}\left(S_{r} \mathbb{I}\right)+\operatorname{div}^{\nabla^{\prime}}\left(\mathcal{A}_{\widehat{W}} \circ T_{r-1}\right) \\
& =(-1)^{r} \nabla^{\prime} S_{r}+\mathcal{A}_{\widehat{W}} \operatorname{div}^{\nabla^{\prime}}\left(T_{r-1}\right)+\sum_{i=1}^{n} \epsilon_{i}\left(\nabla_{Z_{i}}^{\prime} \mathcal{A}_{\widehat{W}}\right) T_{r-1} Z_{i} .
\end{aligned}
$$

Using Codazzi equation

$$
\bar{g}(\bar{R}(X, Y) Z, \widehat{W})=\bar{g}\left(\left(\nabla_{Y}^{\prime} \mathcal{A}_{\widehat{W}}\right) X, Z\right)-\bar{g}\left(\left(\nabla_{X}^{\prime} \mathcal{A}_{\widehat{W}}\right) Y, Z\right),
$$

for any $X, Y, Z \in \Gamma(T \mathcal{F})$ and Lemma 4.1 , we have

$$
\begin{gathered}
\bar{g}\left(\left(\nabla_{Z_{i}}^{\prime} \mathcal{A}_{\widehat{W}}\right) Y, T_{r-1} Z_{i}\right)=\bar{g}\left(\left(\nabla_{Y}^{\prime} \mathcal{A}_{\widehat{W}}\right) Z_{i}, T_{r-1} Z_{i}\right)+\bar{g}\left(\bar{R}\left(Y, Z_{i}\right) T_{r-1} Z_{i}, \widehat{W}\right) \\
=\bar{g}\left(T_{r-1}\left(\nabla_{Y}^{\prime} \mathcal{A}_{\widehat{W}}\right) Z_{i}, Z_{i}\right)+\bar{g}\left(\bar{R}\left(\widehat{W}, T_{r-1} Z_{i}\right) Z_{i}, Y\right),
\end{gathered}
$$


for any $Y \in \Gamma(T \mathcal{F})$. Then applying (4.4) and (4.5) we get

$$
\begin{aligned}
\bar{g}\left(\operatorname{div}^{\nabla^{\prime}}\left(T_{r}\right), Y\right) & =(-1)^{r} \bar{g}\left(\nabla^{\prime} S_{r}, Y\right)+\operatorname{tr}\left(T_{r-1}\left(\nabla_{Y}^{\prime} \mathcal{A}_{\widehat{W}}\right)\right) \\
& +\bar{g}\left(\operatorname{div}^{\nabla^{\prime}}\left(T_{r-1}\right), Y\right)+\bar{g}\left(Y, \sum_{i=1}^{n} \epsilon_{i} \bar{R}\left(\widehat{W}, T_{r-1} Z_{i}\right) Z_{i}\right) .
\end{aligned}
$$

Then, applying (4.6) and (3.6) we get the second equation of the lemma. Finally, (4.3) follows immediately by an induction argument.

Notice that when the ambient manifold is a space form of constant sectional curvature, then $(\bar{R}(\widehat{W}, X) Y)^{\prime}=0$, for each $X, Y \in \Gamma(T \mathcal{F})$. Hence, from Lemma (4.2) we have $\operatorname{div}^{\nabla^{\prime}}\left(T_{r}\right)=0$.

Lemma 4.3. Let $M$ be a screen integrable half-lightlike submanifold of $\bar{M}$ and let $\mathcal{F}$ be a co-dimension three foliation of $S(T M)$. Let $\mathcal{A}_{\widehat{W}}$ be its shape operator, where $\widehat{W}$ is a unit normal vector to $\mathcal{F}$. Let $\left\{Z_{i}\right\}$ be a local field such $\left(\nabla_{X}^{\prime} Z_{i}\right) p=0$, for $i=1, \cdots, n$ and any vector field $X \in \Gamma(T \bar{M})$. Then at $p \in \mathcal{F}$ we have

$$
\begin{aligned}
g\left(\nabla_{Z_{i}}^{\prime} \bar{\nabla}_{\widehat{W}} \widehat{W}, Z_{j}\right) & =g\left(\mathcal{A}_{\widehat{W}}^{2} Z_{i}, Z_{j}\right)-\bar{g}\left(\bar{R}\left(Z_{i}, \widehat{W}\right) Z_{j}, \widehat{W}\right) \\
& -\bar{g}\left(\left(\nabla_{\widehat{W}}^{\prime} \mathcal{A}_{\widehat{W}}\right) Z_{i}, Z_{j}\right)+g\left(\bar{\nabla}_{\widehat{W}} \widehat{W}, Z_{i}\right) g\left(Z_{j}, \bar{\nabla}_{\widehat{W}} \widehat{W}\right) .
\end{aligned}
$$

Proof. Applying $\bar{\nabla}_{Z_{i}}$ to $g\left(\bar{\nabla}_{\widehat{W}} \widehat{W}, Z_{j}\right)$ and $\bar{g}\left(\widehat{W}, \bar{\nabla}_{\widehat{W}} Z_{j}\right)$ in turn and then using the two resulting equations, we have

$$
\begin{aligned}
-\bar{g}\left(\bar{\nabla}_{\widehat{W}} \widehat{W}, \bar{\nabla}_{Z_{i}} Z_{j}\right) & =g\left(\bar{\nabla}_{Z_{i}} \bar{\nabla}_{\widehat{W}} \widehat{W}, Z_{j}\right)+\bar{g}\left(\bar{\nabla}_{Z_{i}} \widehat{W}, \bar{\nabla}_{\widehat{W}} Z_{j}\right) \\
& +\bar{g}\left(\widehat{W}, \bar{\nabla}_{Z_{i}} \bar{\nabla}_{\widehat{W}} Z_{j}\right) .
\end{aligned}
$$

Furthermore, by direct calculations using $\left(\nabla_{X}^{\prime} Z_{i}\right) p=0$ we have

$$
\bar{g}\left(\left(\nabla_{\widehat{W}}^{\prime} \mathcal{A}_{\widehat{W}}\right) Z_{i}, Z_{j}\right)=\bar{g}\left(\bar{\nabla} \widehat{W}_{\widehat{W}}, \overline{Z_{i}} Z_{j}\right)+\bar{g}\left(\widehat{W}, \bar{\nabla}_{\widehat{W}} \bar{Z}_{i} Z_{j}\right),
$$

and hence

$$
\begin{aligned}
g\left(\mathcal{A}_{\widehat{W}}^{2} Z_{i}, Z_{j}\right) & -\bar{g}\left(\bar{R}\left(Z_{i}, \widehat{W}\right) Z_{j}, \widehat{W}\right)-\bar{g}\left(\left(\nabla_{\widehat{W}}^{\prime} \mathcal{A}_{\widehat{W}}\right) Z_{i}, Z_{j}\right) \\
& =g\left(\mathcal{A}_{\widehat{W}}^{2} Z_{i}, Z_{j}\right)-\bar{g}\left(\bar{R}\left(Z_{i}, \widehat{W}\right) Z_{j}, \widehat{W}\right) \\
& -\bar{g}\left(\bar{\nabla}_{\widehat{W}} \widehat{W}, \bar{Z}_{i} Z_{j}\right)-\bar{g}\left(\widehat{W}, \bar{\nabla}_{\widehat{W}} \bar{Z}_{i} Z_{j}\right) \\
& =g\left(\mathcal{A}_{\widehat{W}}^{2} Z_{i}, Z_{j}\right)-\bar{g}\left(\bar{\nabla}_{Z_{i}} Z_{j}, \bar{\nabla}_{\widehat{W}} \widehat{W}\right) \\
& -\bar{g}\left(\bar{\nabla}_{Z_{i}} \bar{\nabla}_{\widehat{W}} Z_{j}, \widehat{W}\right)+\bar{g}\left(\bar{\nabla}_{\left[Z_{i}, \widehat{W}\right]} Z_{j}, \widehat{W}\right) .
\end{aligned}
$$

Now, applying (4.7), the condition at $p$ and the following relations

$$
\bar{\nabla}_{Z_{i}} \widehat{W}=\sum_{k=1}^{n} \epsilon_{k} \bar{g}\left(\bar{\nabla}_{Z_{i}} \widehat{W}, Z_{k}\right) Z_{k}, \quad \bar{\nabla}_{\widehat{W}} Z_{j}=\bar{g}\left(\bar{\nabla}_{\widehat{W}} Z_{j}, \widehat{W}\right) \widehat{W}
$$

and $g\left(\mathcal{A}_{\widehat{W}}^{2} Z_{i}, Z_{j}\right)=-\sum_{k=1}^{n} \epsilon_{k} \bar{g}\left(\bar{\nabla}_{Z_{i}} \widehat{W}, Z_{k}\right) \bar{g}\left(\bar{\nabla}_{Z_{k}} Z_{j}, \widehat{W}\right)$ to the last line of (4.8) and the fact that $S(T M)$ is integrable we get

$$
\begin{aligned}
g\left(\mathcal{A}_{\widehat{W}}^{2} Z_{i}, Z_{j}\right) & -\bar{g}\left(\bar{R}\left(Z_{i}, \widehat{W}\right) Z_{j}, \widehat{W}\right)-\bar{g}\left(\left(\nabla_{\widehat{W}}^{\prime} \mathcal{A}_{\widehat{W}}\right) Z_{i}, Z_{j}\right) \\
& =g\left(\nabla_{Z_{i}}^{\prime} \bar{\nabla}_{\widehat{W}} \widehat{W}, Z_{j}\right)-g\left(\bar{\nabla}_{\widehat{W}} \widehat{W}, Z_{i}\right) g\left(Z_{j}, \bar{\nabla}_{\widehat{W}} \widehat{W}\right),
\end{aligned}
$$

from which the lemma follows by rearrangement.

Notice that, using parallel transport, we can always construct a frame field from the above lemma. 
Proposition 4.4. Let $M$ be a screen integrable half-lightlike submanifold of an indefinite almost contact manifold $\bar{M}$ and let $\mathcal{F}$ be a foliation of $S(T M)$. Then

$$
\begin{aligned}
\operatorname{div}^{\nabla^{\prime}}\left(T_{r} \bar{\nabla}_{\widehat{W}} \widehat{W}\right) & =\bar{g}\left(\operatorname{div}^{\nabla^{\prime}}\left(T_{r}\right), \bar{\nabla}_{\widehat{W}} \widehat{W}\right)+(-1)^{r+1} \widehat{W}\left(S_{r+1}\right) \\
& +(-1)^{r+1}\left(-S_{1} S_{r+1}+(r+2) S_{r+2}\right)-\sum_{i=1}^{n} \epsilon_{i} \bar{g}\left(\bar{R}\left(Z_{i}, \widehat{W}\right) T_{r} Z_{i}, \widehat{W}\right) \\
& +\bar{g}\left(\bar{\nabla}_{\widehat{W}} \widehat{W}, T_{r} \overline{\nabla_{\widehat{W}}} \widehat{W}\right),
\end{aligned}
$$

where $\left\{Z_{i}\right\}$ is a field of frame tangent to the leaves of $\mathcal{F}$.

Proof. From (3.7), we deduce that

$$
\operatorname{div}^{\nabla^{\prime}}\left(T_{r} Z\right)=\bar{g}\left(\operatorname{div}^{\nabla^{\prime}}\left(T_{r}\right), Z\right)+\sum_{i=1}^{n} \epsilon_{i} \bar{g}\left(\nabla_{Z_{i}}^{\prime} Z, T_{r} Z_{i}\right),
$$

for all $Z \in \Gamma(T \mathcal{F})$. Then using (4.9), Lemmas 4.2 and 4.3, we obtain the desired result. Hence the proof.

From Proposition 4.4 we have

Theorem 4.5. Let $M$ be a screen integrable half-lightlike submanifold of an indefinite almost contact manifold $\bar{M}$ and let $\mathcal{F}$ be a co-dimension three foliation of $S(T M)$. Then

$$
\begin{aligned}
\operatorname{div}^{\bar{\nabla}}\left(T_{r} \bar{\nabla}_{\widehat{W}} \widehat{W}\right) & =\bar{g}\left(\operatorname{div}^{\nabla^{\prime}}\left(T_{r}\right), \bar{\nabla}_{\widehat{W}} \widehat{W}\right)+(-1)^{r+1} \widehat{W}\left(S_{r+1}\right) \\
& +(-1)^{r+1}\left(-S_{1} S_{r+1}+(r+2) S_{r+2}\right) \\
& -\sum_{i=1}^{n} \epsilon_{i} \bar{g}\left(\bar{R}\left(Z_{i}, \widehat{W}\right) T_{r} Z_{i}, \widehat{W}\right) .
\end{aligned}
$$

Proof. A proof follows easily from Proposition 4.4 by recognizing the fact that

$$
\begin{aligned}
\operatorname{div}^{\bar{\nabla}}\left(T_{r} \bar{\nabla}_{\widehat{W}} \widehat{W}\right) & =\operatorname{div}^{\nabla^{\prime}}\left(T_{r} \bar{\nabla}_{\widehat{W}} \widehat{W}\right) \\
& -\bar{g}\left(\bar{\nabla}_{\widehat{W}} \widehat{W}, T_{r} \bar{\nabla}_{\widehat{W}} \widehat{W}\right),
\end{aligned}
$$

which completes the proof.

Theorem 4.6. Let $M$ be a screen integrable half-lightlike submanifold of $\bar{M}$ and let $\mathcal{F}$ be a co-dimension three foliation of $S(T M)$. Then,

$$
\begin{aligned}
\operatorname{div}^{\bar{\nabla}}\left(T_{r} \bar{\nabla}_{\widehat{W}} \widehat{W}\right. & \left.+(-1)^{r} S_{r+1} \widehat{W}\right)=\bar{g}\left(\operatorname{div}^{\nabla^{\prime}}\left(T_{r}\right), \bar{\nabla}_{\widehat{W}} \widehat{W}\right) \\
& +(-1)^{r+1}(r+2) S_{r+2}-\sum_{i=1}^{n} \epsilon_{i} \bar{g}\left(\bar{R}\left(Z_{i}, \widehat{W}\right) T_{r} Z_{i}, \widehat{W}\right) .
\end{aligned}
$$

Proof. By straightforward calculations we have

$$
\begin{aligned}
S_{1} & =\operatorname{tr}\left(\mathcal{A}_{\widehat{W}}\right) \\
& =-\sum_{i=1}^{n} \epsilon_{i} \bar{g}\left(\bar{\nabla}_{Z_{i}} \widehat{W}, Z_{i}\right) \\
& =-\sum_{i=1}^{n+1} \epsilon_{i} \bar{g}\left(\bar{\nabla}_{Z_{i}} \widehat{W}, Z_{i}\right) \\
& =-\operatorname{div}^{\bar{\nabla}}(\widehat{W}),
\end{aligned}
$$

where $Z_{n+1}=\widehat{W}$. From this equation we deduce

$$
\operatorname{div}^{\bar{\nabla}}\left(S_{r+1} \widehat{W}\right)=-S_{1} S_{r+1}+\widehat{W}\left(S_{r+1}\right) .
$$

Then from (4.10) and Theorem 4.5 we get our assertion, hence the proof. 
Next, we let $d V$ denote the volume form $\bar{M}$. Then from Theorem 4.6 we have the following

Corollary 4.7. Let $M$ be a screen integrable half-lightlike submanifold of a compact semiRiemannian manifold $\bar{M}$ and let $\mathcal{F}$ be a co-dimension three foliation of $S(T M)$. Then

$$
\begin{aligned}
\int_{\bar{M}} \bar{g}\left(\operatorname{div}^{\nabla^{\prime}}\left(T_{r}\right), \bar{\nabla}_{\widehat{W}} \widehat{W}\right) d V & =\int_{\bar{M}}\left((-1)^{r}(r+2) S_{r+2}\right. \\
& +\sum_{i=1}^{n} \epsilon_{i} \bar{g}\left(\bar{R}\left(Z_{i}, \widehat{W}\right) T_{r} Z_{i}, \widehat{W}\right) d V .
\end{aligned}
$$

Setting $r=0$ in the above corollary we get

Corollary 4.8. Let $M$ be a screen integrable half-lightlike submanifold of a compact semiRiemannian manifold $\bar{M}$ and let $\mathcal{F}$ be a co-dimension three foliation of $S(T M)$ with mean curvatures $S_{r}$. Then for $r=0$ we have

$$
\int_{\bar{M}} 2 S_{2} d V=\int_{\bar{M}} \overline{R i c}(\widehat{W}, \widehat{W}) d V
$$

where $\overline{\operatorname{Ric}}(\widehat{W}, \widehat{W})=\sum_{i=1}^{n} \epsilon_{i} \bar{g}\left(\bar{R}\left(Z_{i}, \widehat{W}\right) \widehat{W}, Z_{i}\right)$.

Notice that the equation in Corollary 4.8 is the lightlike analogue of (3.5) in [2] for co-dimension one foliations on Riemannian manifolds.

Next, we will discuss some consequences of the integral formulas developed so far.

A semi-Riemannian manifold $\bar{M}$ of constant sectional curvature $c$ is called a semiRiemannian space form $[4,6]$ and is denoted by $\bar{M}(c)$. Then, the curvature tensor $\bar{R}$ of $\bar{M}(c)$ is given by

$$
\bar{R}(\bar{X}, \bar{Y}) \bar{Z}=c\{\bar{g}(\bar{Y}, \bar{Z}) \bar{X}-\bar{g}(\bar{X}, \bar{Z}) \bar{Y}\}, \quad \forall \bar{X}, \bar{Y}, \bar{Z} \in \Gamma(T \bar{M}) .
$$

Theorem 4.9. Let $M$ be a screen integrable half-lightlike submanifold of a compact semiRiemannian space form $\bar{M}(c)$ of constant sectional curvature $c$. Let $\mathcal{F}$ be a co-dimension three foliation of its screen distribution $S(T M)$. If $V$ is the total volume of $\bar{M}$, then

$$
\int_{\bar{M}} S_{r} d V=\left\{\begin{array}{cc}
0, & r=2 k+1, \\
c^{\frac{r}{2}\left(\begin{array}{c}
\frac{n}{2} \\
\frac{r}{2}
\end{array}\right) V,} & r=2 k,
\end{array}\right.
$$

for positive integers $k$.

Proof. By setting $\bar{X}=Z_{i}, \bar{Y}=\widehat{W}$ and $Z=T_{r} Z_{i}$ in (4.11) we deduce that

$$
\bar{R}\left(Z_{i}, \widehat{W}\right) T_{r} Z_{i}=-c g\left(Z_{i}, T_{r} Z_{i}\right) \widehat{W}
$$

Then substituting this equation in Corollary 4.7 we obtain

$$
\int_{\bar{M}} \bar{g}\left(\operatorname{div}^{\nabla^{\prime}}\left(T_{r}\right), \bar{\nabla}_{\widehat{W}} \widehat{W}\right) d V=\int_{\bar{M}}\left((-1)^{r}(r+2) S_{r+2}-c \operatorname{tr}\left(T_{r}\right)\right) d V .
$$

Since $\bar{M}$ is of constant sectional curvature $c$, then Lemma 4.2 implies that $T_{r}=0$ for any $r$ and hence the above equation simplifies to

$$
(r+2) \int_{\bar{M}} S_{r+2} d V=c(n-r) \int_{\bar{M}} S_{r} d V
$$

Since $S_{1}=-\operatorname{div}^{\bar{\nabla}}(\widehat{W})$ and that $\bar{M}$ is compact, then $\int_{\bar{M}} S_{1} d V=0$. Using this fact together with (4.13), mathematical induction gives $\int_{\bar{M}} S_{r} d V=0$ for all $r=2 k+1$ (i.e., $r$ odd). 
For $r$ even we will consider $r=2 m$ and $n=2 l$ (i.e., both $M$ and $\bar{M}$ are odd dimensional). With these conditions, (4.13) reduces to

$$
\int_{\bar{M}} S_{2 m+2} d V=c \frac{l-m}{1+m} \int_{\bar{M}} S_{2 m} d V .
$$

Now setting $m=0,1, \cdots$ and $S_{0}=1$ in (4.14) we obtain

$$
\int_{\bar{M}} S_{2} d V=c l V, \quad \int_{\bar{M}} S_{4} d V=c^{2} \frac{(l-1) l}{2} V
$$

and more generally

$$
\int_{\bar{M}} S_{2 k} d V=c^{k} \frac{(l-k+1)(l-k+2)(l-k+3) \cdots l}{k !} V .
$$

Hence, our assertion follows from 4.15, which completes the proof.

Next, when $\bar{M}$ is Einstein i.e., $\overline{\text { Ric }}=\mu \bar{g}$ we have the following.

Theorem 4.10. Let $M$ be a screen integrable half-lightlike submanifold of an Einstein compact semi-Riemannian manifold $\bar{M}$. Let $\mathcal{F}$ be a co-dimension three foliation of its screen distribution $S(T M)$ with totally umbilical leaves. If $V$ is the total volume of $\bar{M}$, then

$$
\int_{\bar{M}} S_{r} d V=\left\{\begin{array}{cc}
0, & r=2 k+1, \\
\left(\frac{\mu}{n}\right)^{\frac{n}{2}}\left(\begin{array}{c}
\frac{n}{2} \\
\frac{r}{2}
\end{array}\right) V, & r=2 k,
\end{array}\right.
$$

for positive integers $k$.

Proof. Suppose that $\mathcal{A}_{\widehat{W}}=\frac{1}{n} S_{r} \mathbb{I}$. Then by direct calculations using the formula for $T_{r}$ we derive $T_{r}=(-1)^{r+1} \frac{(n-r)}{n} S_{r} \mathbb{I}$. Then, under the assumptions of the theorem we obtain $\overline{\operatorname{Ric}}\left(\widehat{W}, \bar{\nabla}_{\widehat{W}} \widehat{W}\right)=0$ and $\frac{n}{\operatorname{Ric}}(\widehat{W}, \widehat{W})=\mu$ and hence, Corollary 4.7 reduces to

$$
n(r+2) \int_{\bar{M}} S_{r+2} d V=\lambda(n-r) \int_{\bar{M}} S_{r} d V .
$$

Notice that (4.17) is similar to (4.13) and hence following similar steps as in the previous theorem we get $\int_{\bar{M}} S_{r} d V=0$ for $r$ odd and for $r$ even we get

$$
\int_{\bar{M}} S_{2 k} d V=\left(\frac{\mu}{n}\right)^{k} \frac{(l-k+1)(l-k+2)(l-k+3) \cdots l}{k !} V
$$

which complete the proof.

\section{Screen umbilical half-lightlike submanifolds}

In this section we consider totally umbilical half-lightlike submanifolds of semi-Riemannian manifold, with a totally umbilical screen distribution and thus, give a generalized version of Theorem 4.3.7 of [6] and its Corollaries, via Newton transformations of the operator $A_{N}$.

A screen distribution $S(T M)$ of a half-lightlike submanifold $M$ of a semi-Riemannian manifold $\bar{M}$ is said to be totally umbilical [6] if on any coordinate neighborhood $\mathcal{U}$ there exist a function $K$ such that

$$
C(X, P Y)=K g(X, P Y), \quad \forall X, Y \in \Gamma(T M) .
$$

In case $K=0$, we say that $S(T M)$ is totally geodesic. Furthermore, if $S(T M)$ is totally umbilical then by straightforward calculations we have

$$
A_{N} X=P X, \quad C(E, P X)=0, \quad \forall X \in \Gamma(T M) .
$$


Let $\left\{E, Z_{i}\right\}$, for $i=1, \cdots, n$, be a quasi-orthonormal frame field of $T M$ which diagonalizes $A_{N}$. Let $l_{0}, l_{1}, \cdots, l_{n}$ be the respective eigenvalues (or principal curvatures). Then as before, the $r$-th mean curvature $S_{r}$ is given by

$$
S_{r}=\sigma_{r}\left(l_{0}, \cdots, l_{n}\right) \text { and } S_{0}=1 .
$$

The characteristic polynomial of $A_{N}$ is given by

$$
\operatorname{det}\left(A_{N}-t \mathbb{I}\right)=\sum_{\alpha=0}^{n}(-1)^{\alpha} S_{r} t^{n-\alpha},
$$

where $\mathbb{I}$ is the identity in $\Gamma(T M)$. The normalized $r$-th mean curvature $H_{r}$ of $M$ is defined by $\left(\begin{array}{l}n \\ r\end{array}\right) H_{r}=S_{r} \quad$ and $\quad H_{0}=1$. The Newton transformations $T_{r}: \Gamma(T M) \rightarrow \Gamma(T M)$ of $A_{N}$ are given by the inductive formula

$$
T_{0}=\mathbb{I}, \quad T_{r}=(-1)^{r} S_{r} \mathbb{I}+A_{N} \circ T_{r-1}, \quad 1 \leq r \leq n .
$$

By Cayley-Hamiliton theorem, we have $T_{n+1}=0$. Also, $T_{r}$ satisfies the following properties.

$$
\begin{aligned}
\operatorname{tr}\left(T_{r}\right) & =(-1)^{r}(n+1-r) S_{r}, \\
\operatorname{tr}\left(A_{N} \circ T_{r}\right) & =(-1)^{r}(r+1) S_{r+1}, \\
\operatorname{tr}\left(A_{N}^{2} \circ T_{r}\right) & =(-1)^{r+1}\left(-S_{1} S_{r+1}+(r+2) S_{r+2}\right), \\
\operatorname{tr}\left(T_{r} \circ \nabla_{X} A_{N}\right) & =(-1)^{r} X\left(S_{r+1}\right),
\end{aligned}
$$

for all $X \in \Gamma(T M)$.

Proposition 5.1. Let $(M, g)$ be a totally umbilical half-lightlike submanifold of a semiRiemannian manifold $\bar{M}$ of constant sectional curvature $c$. Then

$$
\begin{aligned}
g\left(\operatorname{div}^{\nabla}\left(T_{r}\right), X\right) & =(-1)^{r-1} \lambda(X) E\left(S_{r}\right)-\tau(X) \operatorname{tr}\left(A_{N} \circ T_{r-1}\right) \\
& -c \lambda(X) \operatorname{tr}\left(T_{r-1}\right)+g\left(\operatorname{div}^{\nabla}\left(T_{r-1}\right), A_{N} X\right)+g\left(\left(\nabla_{E} A_{N}\right) T_{r-1} E, X\right) \\
& +\sum_{i=1}^{n} \epsilon_{i}\left\{-\lambda(X) B\left(Z_{i}, A_{N}\left(T_{r-1} Z_{i}\right)\right)\right. \\
& \left.+\varepsilon \tau\left(Z_{i}\right) C\left(X, T_{r-1} Z_{i}\right)\left\{\rho(X) D\left(Z_{i}, T_{r-1} Z_{i}\right)-\rho\left(Z_{i}\right) D\left(X, T_{r-1} Z_{i}\right)\right\}\right\},
\end{aligned}
$$

for any $X \in \Gamma(T M)$.

Proof. From the recurrence relation (5.3), we derive

$$
\begin{aligned}
g\left(\operatorname{div}^{\nabla}\left(T_{r}\right), X\right) & =(-1)^{r} P X\left(S_{r}\right)+g\left(\left(\nabla_{E} A_{N}\right) T_{r-1} E, X\right) \\
& +g\left(\operatorname{div}^{\nabla}\left(T_{r-1}\right), A_{N} X\right)+\sum_{i=1}^{n} \epsilon_{i} g\left(\left(\nabla_{Z_{i}} A_{N}\right) T_{r-1} Z_{i}, X\right),
\end{aligned}
$$

for any $X \in \Gamma(T M)$. But

$$
\begin{aligned}
g\left(\left(\nabla_{Z_{i}} A_{N}\right) T_{r-1} Z_{i}, X\right) & =g\left(T_{r-1} Z_{i},\left(\nabla_{Z_{i}} A_{N}\right) X\right)+g\left(\nabla_{Z_{i}} A_{N}\left(T_{r-1} Z_{i}\right), X\right) \\
& -g\left(\nabla_{Z_{i}}\left(A_{N} X\right), T_{r-1} Z_{i}\right)+g\left(A_{N}\left(\nabla_{Z_{i}} X\right), T_{r-1} Z_{i}\right) \\
& -g\left(A_{N}\left(\nabla_{Z_{i}} T_{r-1} Z_{i}\right), X\right),
\end{aligned}
$$

for all $X \in \Gamma(T M)$.

Then applying (2.9) to (5.9) while considering the fact that $A_{N}$ is screen-valued, we get

$$
g\left(\left(\nabla_{Z_{i}} A_{N}\right) T_{r-1} Z_{i}, X\right)=g\left(T_{r-1} Z_{i},\left(\nabla_{Z_{i}} A_{N}\right) X\right)-\lambda(X) B\left(Z_{i}, A_{N}\left(T_{r-1} Z_{i}\right)\right) .
$$


Furthermore, using (2.15) and (4.11), the first term on the right hand side of (5.10) reduces to

$$
\begin{aligned}
g\left(T_{r-1} Z_{i},\right. & \left.\left(\nabla_{Z_{i}} A_{N}\right) X\right)=-c \lambda(X) g\left(Z_{i}, T_{r-1} Z_{i}\right)+g\left(\left(\nabla_{X} A_{N}\right) Z_{i}, T_{r-1} Z_{i}\right) \\
& -\tau(X) C\left(Z_{i}, T_{r-1} Z_{i}\right)+\varepsilon \tau\left(Z_{i}\right) C\left(X, T_{r-1} Z_{i}\right)\left\{\rho(X) D\left(Z_{i}, T_{r-1} Z_{i}\right)\right. \\
& \left.-\rho(X) D\left(X, T_{r-1} Z_{i}\right)\right\},
\end{aligned}
$$

for any $X \in \Gamma(T M)$. Finally, replacing (5.11) in (5.10) and then put the resulting equation in (5.8) we get the desired result.

Next, from Proposition 5.1 we have the following.

Theorem 5.2. Let $(M, g)$ be a half-lightlike submanifold of a semi-Riemannian manifold $\bar{M}(c)$ of constant curvature $c$, with a proper totally umbilical screen distribution $S(T M)$. If $M$ is also totally umbilical, then the $r$-th mean curvature $S_{r}$, for $r=0,1, \cdots, n$, with respect to $A_{N}$ are solution of the following differential equation

$$
E\left(S_{r+1}\right)-\tau(E)(r+1) S_{r+1}-c(-1)^{r}(n+1-r) S_{r}=\mathcal{H}_{1}(r+1) S_{r+1} .
$$

Proof. Replacing $X$ with $E$ in the Proposition 5.1 and then using (2.16) and (5.2) we obtain, for all $r=0,1, \cdots, n$,

$$
E\left(S_{r+1}\right)-(-1)^{r} \tau(E) \operatorname{tr}\left(A_{N} \circ T_{r}\right)-c(-1)^{r} \operatorname{tr}\left(T_{r}\right)=(-1)^{r} \mathcal{H}_{1} \operatorname{tr}\left(A_{N} \circ T_{r}\right),
$$

from which the result follows by applying (5.4) and (5.5).

Corollary 5.3. Under the hypothesis of Theorem 5.2, the induced connection $\nabla$ on $M$ is a metric connection, if and only if, the $r$-th mean curvature $S_{r}$ with respect to $A_{N}$ are solution of the following equation

$$
E\left(S_{r+1}\right)-\tau(E)(r+1) S_{r+1}-c(-1)^{r}(n+1-r) S_{r}=0 .
$$

Also the following holds.

Corollary 5.4. Under the hypothesis of Theorem 5.2, $\bar{M}(c)$ is a semi-Euclidean space, if and only if, the $r$-th mean curvature $S_{r}$ with respect to $A_{N}$ are solution of the following equation

$$
E\left(S_{r+1}\right)-\tau(E)(r+1) S_{r+1}=\mathcal{H}_{1}(r+1) S_{r+1} .
$$

Notice that Theorem 5.2 and Corollary 5.3 are generalizations of Theorem 4.3.7 and Corollary 4.3.8, respectively, given in [6].

Acknowledgment. This work is based on the research supported wholly by the National Research Foundation of South Africa (Grant Numbers: 95931 and 106072).

\section{References}

[1] K. Andrzejewski, W. Kozlowski and K. Niedzialomski, Generalized Newton transformation and its applications to extrinsic geometry, Asian J. Math. 20 (2), 293-322, 2016.

[2] K. Andrzejewski and Pawel G. Walczak, The Newton transformation and new integral formulae for foliated manifolds, Ann. Glob. Anal. Geom. 37 (2), 103-111, 2010.

[3] C. Calin, Contributions to geometry of CR-submanifold, Ph.D. thesis, University of Iasi (Romania), 1998.

[4] K.L. Duggal and A. Bejancu, Lightlike submanifolds of semi-Riemannian manifolds and applications, Mathematics and Its Applications, Kluwer Academic Publishers, 1996.

[5] K.L. Duggal and B. Sahin, Screen conformal half-lightlike submanifolds, Int. J. Math. Math. Sci. 2004 (68), 3737-3753, 2004. 
[6] K.L. Duggal and B. Sahin, Differential geometry of lightlike submanifolds. Frontiers in Mathematics, Birkhäuser Verlag, Basel, 2010.

[7] D.N. Kupeli, Singuler semi-Riemannian geometry, Mathematics and Its Applications, Vol. 366, Kluwer Academic Publishers, 1996.

[8] F. Massamba, Totally contact umbilical lightlike hypersurfaces of indefinite Sasakian manifolds, Kodai Math. J. 31, 338-358, 2008.

[9] F. Massamba, On semi-parallel lightlike hypersurfaces of indefinite Kenmotsu manifolds, J. Geom. 95, 73-89, 2009.

[10] F. Massamba and S. Ssekajja, Some remarks on quasi generalized CR-null geometry in indefinite nearly cosymplectic manifolds, Int. J. Math. Math. Sci. Art. ID 9613182, $10 \mathrm{pp}, 2016$.

[11] E. Yasar, A.C. Coken, and A. Yucesan, Lightlike hypersurfaces in semi-Riemannian manifold with semi-symmetric non-metric connection, Math. Scand. 102 (2), 253264, 2008. 